\title{
Astha Srivastava* and Ankur Srivastava* Economic Analysis of Accident Law: A New Liability Rule that Induces Socially Optimal Behaviour in Case of Limited Information
}

https://doi.org/10.1515/rle-2019-0049

Published online November 19, 2020

Abstract: In accident law, we seek a liability rule that will induce both the parties to adopt socially optimal levels of precaution. Economic analysis, however, shows that none of the commonly used liability rules induce both parties to adopt optimal levels, if courts have access only to 'Limited Information' on. In such a case, it has also been established (K. (2006). Efficiency of liability rules: a reconsideration. J. Int. Trade Econ. Dev. 15: 359-373) that no liability rule based on cost justified untaken precaution as a standard of care can be efficient. In this paper, we describe a two-step liability rule: the rule of negligence with the defence of relative negligence. We prove that this rule has a unique Nash equilibrium at socially optimal levels of care for the non-cooperative game, and therefore induces both parties to adopt socially optimal behaviour even in case of limited information.

Keywords: Tort law, economic analysis of law, liability rules, Hand's rule, limited information

\section{Introduction}

Accident laws aim to provide liability rules that apportion the costs of an accident between the injurer and the victim. From an economic perspective, the rule should induce the parties to adopt levels of precaution that minimise the total social cost of the accident. A central feature of most of the commonly adopted liability rules is 'negligence', that is, whether or not an actor complies with a 'standard of care'. In any suit, courts are required to determine whether a party is negligent, which in turn forms the basis for the apportionment of loss. A study of how such a

*Corresponding authors: Astha Srivastava, ICFAI Law School, Hyderabad, India, E-mail: astha.davv@gmail.com. https://orcid.org/0000-0003-4297-4375; and Ankur Srivastava, Master of Business Law Deputy Chief Vigilance Officer, Government of India, India, E-mail:a.anksri@gmail.com 
determination is made in courtroom settings and its impact on the optimality of the outcome is therefore of considerable importance.

Holmes, in his book "The Common Law" takes the position that courts determine negligence through a two-step process. First, they establish the (external and objective) standard of care for the accident at hand; then they measure the actor's conduct against it (as reported in Grady 1989). In the same paper, however, Grady points out that this approach is rarely adopted by courts in real life. In the adversarial system, courts limit themselves to an assessment of plaintiff's (the victim's) allegation of the untaken precautions available to the defendant. If the potential benefit of such a precaution exceeds the cost thereof (that is, the said untaken precaution is cost justified), the defendant is held negligent. This method was indeed adopted by Judge Learned Hand in the celebrated case of United States v Carroll Towing Co. ${ }^{1}$ and has come to be known as the Hand Rule.

Mathematical formalism for economic analysis of liability rules under tort laws was developed by Brown in his seminal paper (Brown 1973). In this paper, Brown considered the case of bilateral precaution, where both the injurer and the victim can take precaution to abate the possibility of harm, and the precautions taken by injurer and victim are strategic substitutes. Brown also adopted the Hand rule (interpreted in an incremental sense, i.e. comparing marginal benefit to marginal cost) as the standard of care. It was established that

(i) if courts have access to complete information about the range of accident precaution (i.e. they can determine the marginal benefit and cost of additional precaution at all levels of precaution), and

(ii) they adopt the Hand rule as the standard of care,

then all of the negligence-based liability rules induce both parties to adopt socially optimal behaviour. A complete characterisation of efficient liability rules has been presented in Jain and Singh (2002). This is possible because courts can determine the optimal levels of precaution using complete information and can test the actual precaution taken by parties with this optimal level to determine negligence. As is easily seen, this is akin to the two-step process theorised by Holmes.

However, prohibitively high information costs and other factors make it unlikely that courts will have access to such complete information in a real world scenario. As Grady (1989) points out, this is also not the way that courts function.

1 159 F.2d 169 (2d Cir. 1947). 
Brown (1973) also analysed liability rules with a more realistic assumption that courts do not have access to complete information, but can assess the marginal benefits and costs of taking additional care by either party at the current levels of care. This, Brown terms as the 'Limited Information' (LI) case, and it is essentially the same as the method of untaken precaution suggested by Grady. Brown proved that in such a case, none of the negligence-based liability rules have a Nash equilibrium at socially optimal levels and therefore they do not induce the parties to adopt the optimal level of precaution (a more rigorous proof of this conclusion is presented in S. K. Jain 2009). Brown had presented the rule of "relative negligence" as a possible liability rule for a socially optimal outcome in an LI regime. However, there is an error in this analysis and even the relative negligence rule will not lead to a socially optimal outcome in the case of limited information (Assaf 1984).

A liability rule that would lead to socially optimal outcomes through the routine adversarial system of court proceedings would stand on sound economic footing and therefore would be the rule of choice under tort laws (Of course there are other factors in judicial process that also hinder an efficient outcome, for example, uncertainity in assessment of liability (Shavell and Kaplow 1994) or damages (Shavell and Kaplow 1996). However, we ignore these irritants for the purpose of our analysis in this paper). The pessimistic result of Brown has therefore been discussed and analysed in several subsequent papers (see Diamond 1974 and Green 1976), and attempts have been made to identify strategies that would induce the parties to adopt optimal levels of precaution even under the limited information case. Cooter et al. have examined the role of judicial precedents under the limited information regime (Cooter et al. 1979) and hold that with negligence based liability rules, courts can tend towards optimal levels of standard of care by successive improvements in the standards set by precedents.

This solution, however, is beset with two complications. First, it assumes that courts can obtain information regarding the marginal benefits and costs of both parties at the current standard of care. The current standard of care may be different from the current level of care actually taken by the parties. Whether this information can be obtained in a litigation is doubtful, because for a party to prove the marginal benefit at the current standard of care will require the assessment of marginal benefits from the current level of care actually taken to the current standard of care, which effectively implies obtaining complete information about a substantial portion of the probability curve.

Second, this methodology requires repeated litigation on the same matter. Considering that no two cases will be on exactly the same facts or even on the same nature of tortuous act, it is likely that the precedents will present a confusing 
picture which will diminish their utility for the purpose of the evolution of standards of care.

A similar approach of using an evolutionary standard of care has been presented in Ott and Schafer (1997) which, however, is restricted only to cases of unilateral precaution. Another alternative method has been suggested in Guttel (2007), which proposes to allow a party to claim restitution in respect of all costjustified precautions. As we show using the mathematical model of Brown (in Appendix II), this rule does not have a Nash equilibrium at the socially optimal level. Further, it is clear that this approach too requires courts to ascertain all precautions already taken and so does not fall within the ambit of the limited information case, as described in Brown (1973). ${ }^{2}$

The search for an efficient liability rule in a limited information regime, therefore, still continues more than 40 years after the initial results of Brown. Jain (2006) has in fact shown that any negligence-based liability rule, where the standard of care adopted is based on cost justified untaken precaution will not lead to an efficient outcome.

In this study, it has been our endeavour to find an efficient liability rule under limited information. Learning from the earlier results, we propose a two-step liability rule: the rule of negligence with the defence of relative negligence. We will prove that this rule has a unique Nash equilibrium at the socially optimal levels of care for the non-cooperative game, and therefore induces both parties to adopt socially optimal behaviour.

The organisation of this paper is as follows: In Section 2, we briefly lay down the mathematical formulation adopted in economic analysis of accident law, including a mathematical representation of some common liability rules. In Section 3, we show two different approaches commonly adopted to determine negligence ("the standard of care") in cases where only limited information is available. In Section 4, we define the proposed liability rule, and prove that this rule has a unique Nash equilibrium at socially optimal levels even under a limited information regime, and therefore leads to efficient outcomes. Inferences from the results obtained are presented in Section 5.

\footnotetext{
2 Imposing liability for harm has also been compared with ex-ante regulation of potentially harmful activities (Rose-Ackerman 1991). While there can be merit in adopting regulation on the grounds of better technical capability of regulating agencies, there will still be cases where harm occurs and so recourse to a judicial determination of negligence is still required. Even if otherwise, Shavell (2013) proves that there is a fundamental enforcement cost advantage of a negligence rule over regulation.
} 


\section{Mathematical Formulation of Accident Law}

Broadly following the formulation developed in Brown (1973), we consider a case where a party 'I' (the injurer) engages in an activity that may cause loss to property of ' $\mathrm{V}$ ' (the victim). It is assumed that there is a possibility of bilateral precaution, that is, both the parties can take precautions to reduce the probability of such a loss occurring.

We denote the level of care adopted by injurer as $X$, and the level of care adopted by the victim as $Y$.

Let the cost of taking each additional unit of precaution by injurer be $w_{x}$ and that of victim be $w_{y}$. In this analysis, these (marginal) costs are assumed to be fixed for any level of care. However, the results of our analysis will be true even when these costs are (sufficiently well behaved) functions of the respective levels of care.

Probability of accident is denoted as $\{1-p(X, Y)\}$, which is a function of $X$ and $Y$. The loss, in the event of an accident happening, is also assumed to be a fixed amount ' $A$ '.

With these assumptions, the total social cost (TSC) at a certain level of care taken by the parties $(X, Y)$ shall be

$$
T S C=w_{x} \cdot X+w_{y} \cdot Y+\{1-p(X, Y)\} \cdot A
$$

An economically efficient outcome will result when TSC is minimised. The corresponding levels of care are denoted as socially optimal levels $\left(X^{*}, Y^{*}\right)$. We obtain the following important results:

$$
\begin{gathered}
w_{x}=p_{x}\left(X^{*}, Y^{*}\right) \cdot A \text { and }, \\
w_{y}=p_{y}\left(X^{*}, Y^{*}\right) \cdot A
\end{gathered}
$$

Here, $p_{x}$ and $p_{y}$ are the marginal reduction in probability of occurrence of loss (i.e. the marginal benefit of additional care), for changes in $X$ and $Y$ respectively, while keeping the other operating parameter constant.

We also adopt the full cost response function described in Brown's paper, $X^{f}(Y)$. This is the value of $X$ that will minimise TSC for any given value of $Y$. Correspondingly, $Y^{f}(X)$ is the value of $Y$ that will minimise TSC for any given value of $X$.

In any liability rule, the loss is apportioned between the injurer and the victim. We represent this as a loss function $L_{x}(X, Y) \& L_{y}(X, Y)$ for injurer and victim respectively. The parties seek to minimize their own total cost (apportioned loss + cost of taking care). For example, for injurer the total cost is $\left.L_{X}(X, Y) .\{1-p(X, Y)\} A+w_{x} \cdot X\right)$.

The loss function of injurer is presented for the case of simple negligence as an example 


$$
L_{X}(X, Y)=\left\{\frac{1: \text { if } X<X^{\sim}}{0 \text { Otherwise }}\right.
$$

where $X^{\sim}$ is the mandated level of due care for the injurer. Similarly, the loss function for the case of relative negligence is

$$
L_{x}(X, Y)=\left\{\begin{array}{l}
1: \text { if } \frac{p_{X}(X, Y)}{p_{y}(X, Y)}>\frac{w_{x}}{w_{y}} \\
0 \text { Otherwise }
\end{array}\right.
$$

where $\frac{p_{X}(X, Y)}{p_{y}(X, Y)}$ is a measure of relative negligence of injurer as compared with that of victim.

\section{Comparison of Methods to Determine Standards of Care: Hand Rule vs. Relative Negligence}

Under negligence-based liability rules, the party which is found negligent has to bear the loss caused due to accident. For a socially optimal outcome, courts must set the respective standards of care as $\left(X^{*}, Y^{*}\right)$ for determining negligence. However, these optimal levels are not known to the courts a priori and must be determined from the facts of the case. As discussed earlier, in the limited information scenario, courts do not have access to all the information required to correctly determine these standards. The method adopted by courts to determine the negligence of either party is of crucial importance as it will determine the level of care actually adopted by the parties.

The first approach is that of the Hand rule, which seeks to assess whether, for one of the parties, the marginal benefit of taking additional precaution exceeds the marginal cost of taking such precaution at the current levels of care. In other words, a party is found negligent if it did not take additional precaution that would have been cost justified at the current levels of care. It is easily seen that this approach is identical to the principle of cost-justified untaken precaution described earlier.

Mathematically, if at the time of an accident, the level of care by the parties is $\left(X^{o}, Y^{o}\right)$ (which may or may not be the same as $\left(X^{*}, Y^{*}\right)$ ), adopting the simple negligence liability rule, which requires determination of the negligence of injurer only, the injurer will be found negligent under the Hand rule if:

$$
w_{x}<p_{x}\left(X^{o}, Y^{o}\right) \cdot A
$$

A second approach is that of relative negligence, which seeks to determine which of the two parties is more negligent. Mathematically, the injurer is negligent if: 


$$
\frac{p_{x}\left(X^{o}, Y^{o}\right)}{p_{y}\left(X^{o}, Y^{o}\right)}>\frac{w_{x}}{w_{y}}
$$

This is based on the concept of imposing the burden of care on the "least cost avoider". This approach has also been favoured in (Calabresi 1970).

Other liability rules have also been explored elsewhere (Rubinfeld 1987). However, as stated earlier, prior literature shows that none of these methods leads to a Nash equilibrium at the socially optimal level of care $\left(X^{*}, Y^{*}\right)$, and therefore, each fails to induce the parties to adopt these optimal levels. The logical way forward would be to look for an amalgamation of these two approaches for assessment of negligence.

\section{The Rule of Negligence with Defence of Relative Negligence}

We adopt the following two-step rule for assessing negligence

Criterion1: Check whether additional precaution by injurer is cost justified at current levels of care $\left(X^{0}, Y^{\circ}\right)$. (This is the Hand rule for assessment of negligence)

$$
w_{x} \geq p_{x}\left(X^{o}, Y^{o}\right) \cdot A
$$

Criterion 2: Check whether victim is relatively more negligent at current levels of care. (This is the defence of relative negligence)

$$
\frac{p_{y}\left(X^{o}, Y^{o}\right)}{p_{x}\left(X^{o}, Y^{o}\right)} \geq \frac{w_{y}}{w_{x}}
$$

If either of the above criteria is satisfied, victim bears the whole loss, otherwise injurer bears the loss.

It is evident that for these criteria, only the probability function and its derivatives at $\left(X^{o}, Y^{o}\right)$, in other words, only limited information as defined in Brown (1973), is needed. We seek to prove that this negligence-based liability rule has a unique Nash Equilibrium at the socially optimal levels of care $\left(X^{*}, Y^{*}\right)$.

We assume, $p_{x y}<0$ in the whole domain of $X$ and $Y$ (see Brown 1973 for a justification). We shall also make an additional assumption as follows:

$$
\frac{\partial}{\partial x}\left(p_{x} / p_{y}\right)<0 \text { for all } X \text { and } Y
$$


where $\frac{\partial}{\partial x}$ measures the marginal change of any function for an additional amount of $X$, while keeping $Y$ constant. Correspondingly, we also assume

$$
\frac{\partial}{\partial y}\left(p_{y} / p_{x}\right)<0 \text { for all } X \text { and } Y
$$

Assumptions (5) and (6) are mathematical representations of our expectation that any additional care taken by one party would always reduce its relative negligence. It is indeed counterintuitive to imagine a situation where additional precaution by one party causes its relative negligence to increase. We shall show in Appendix I, the mathematical implication of these additional assumptions.

\subsection{Proof of Nash Equilibrium}

1. The immediate consequence of $p_{x y}<0$ is that whenever $Y$ increases, the corresponding value of $X^{f}$ decreases. A similar statement can be made for $Y^{f}$.

2. We first prove that $\left(X^{*}, Y^{*}\right)$ is a Nash equilibrium.

a. At this point, both of the following equations are satisfied

$$
\begin{gathered}
w_{x}=p_{x}(X, Y) \cdot A \\
\text { And } \frac{p_{y}(X, Y)}{p_{x}(X, Y)}=\frac{w_{y}}{w_{x}}
\end{gathered}
$$

b. As both criteria are satisfied, victim bears the loss.

\section{Analysis of victim's strategy}

c. If $X$ remains unchanged and the victim increases his level of care $(Y)$, the Right Hand Side of Eq. (7) would decrease (as $p_{x y}<0$ ) and so criterion 1 would still be satisfied. In this scenario the victim will continue to be liable to bear the loss and therefore the full cost curve shall be applicable for the victim. We know that the minimum of victim's full cost curve for $\mathrm{X}=X^{*}$, $Y^{f}\left(X^{*}\right)$ is same as $Y^{*}$. Hence, there is no incentive for victim to increase his level of care beyond $Y^{*}$.

d. If the victim decreases his level of care, the ratio $p_{y} / p_{x}$ increases (by our assumption in Eq. (6)) and continues to satisfy criterion 2, making the victim liable. As in the previous case, the minimum for victim's cost is at $Y=Y^{*}$. Hence at $X=X^{*}$, victim has no incentive to move away from $Y=Y^{*}$ Analysis of injurer's strategy

e. For same $Y$, injurer is not liable for accident costs anyway, and so has no incentive to increase his level of care, which will only add to his cost of care. 
f. A decrease in his level of care will cause injurer to fail on both the criteria, and will therefore shift from no liability curve to full liability curve. It is evident that the cost on the full liability curve would be higher for the injurer.

Hence at $Y=Y^{*}$, injurer has no incentive to move away from $X=X^{*}$

Thus, a Nash equilibrium exists at $\left(X^{*}, Y^{*}\right)$.

3. We now prove that no other Nash equilibrium exists.

a. Consider $X>X^{*}$. We can show that for any value of $Y$, at least one of the two criteria is always satisfied. Hence, victim would always bear the loss and therefore his optimal strategy is $Y=Y^{f}(X)$. Correspondingly, the optimal strategy for $X$ is $X^{\#}=X^{f}\left(Y^{f}(X)\right)$. It is easy to see that for all $X>X^{*}, X^{\#}<X^{*}$. Hence, no $X>X^{*}$ is a Nash equilibrium.

b. Similarly, if $X<X^{*}$, victim can always choose a $Y$ less than $Y^{*}$, where injurer would bear the loss. At this point, injurer operates at full cost curve and its cost minimum will be at $X=X^{f}(Y)$. We know that for all $Y$ less than $Y^{*}$, $X^{f}(Y)$ is greater than $X^{*}$, hence no Nash equilibrium exists.

Thus, our main result:

The proposed 2-step liability rule of negligence with the defence of relative negligence, has a unique Nash Equilibrium at the socially optimal levels of care.

The logic behind this rule is illuminating. At the socially optimal level of care, the rule of cost justified untaken precaution (Hand rule) provides no incentive to the victim to increase her level of care, but induces the victim to reduce her level of care as that has the effect of increasing $p_{x}$ which makes the injurer liable to bear the loss (this possibility of opportunistic inefficient care has been discussed in Guttel 2007). On the other hand, the rule of relative negligence provides no incentive for the victim to decrease her level of care, but provides an incentive to increase her level of care as it reduces her relative negligence below the threshold, thereby making injurer liable for the loss (this is a direct outcome of our assumptions in Eq.(5) and (6)). Both the rules, used in conjunction, act in "opposite directions" so that each destroys the possible incentives provided by the other to move away from the optimal level. As a result, a Nash equilibrium emerges at the socially optimal level.

\section{Conclusion}

In realistic situations, it is very difficult for courts to find out the socially optimal levels of care in the usual process of litigation. However, the limited information 
regarding marginal benefits and costs of care by the parties at current levels of care is more easily determined. For example, the victim may provide evidence to demonstrate the benefits and cost of an additional care that the injurer might have taken. Similarly, the injurer may establish the marginal costs and benefits of an additional care by the injured. This way, in the process of litigation the courts can become aware of the marginal costs and benefits of care for both of the parties, i.e. $p_{x}, p_{y}, w_{x}$ and $w_{y}$ at the currently adopted levels of care.

The utility of the proposed rule lies in its application in such real-world scenarios. If this two-step rule is adopted by courts to determine the negligence or otherwise of the parties, both of the parties would be induced to adopt the socially optimal behaviour. The proposed two-step rule can therefore be the liability rule of choice in tort litigations.

\section{Appendix I}

\section{Mathematical implication of assumptions in Eqs. (5) and (6)}

$$
\begin{aligned}
& \frac{\partial}{\partial y}\left(p_{y} / p_{x}\right)<0 \text { implies } \\
& p_{y y} \cdot p_{x}-p_{x y} \cdot p_{y}<0 \\
& \text { i.e } \frac{p_{y y}}{p_{y}}<\frac{p_{x y}}{p_{x}} \\
& \text { or, } p_{x y}>\frac{p_{x}}{p_{y}} \cdot p_{y y}
\end{aligned}
$$

Similarly, from Eq. (5), we have

$$
p_{x y}>\frac{p_{y}}{p_{x}} \cdot p_{x x}
$$

We know that in bilateral precaution, additional care by one party changes the probability of accident as well as the marginal benefit of additional care for both the parties. The import of the above two relations is that for any additional care taken by one party, the percentage reduction in marginal benefit for that party is more than the percentage reduction in marginal benefit of the other party. In other words, additional care by one party has a more direct impact on its own marginal benefits than on the marginal benefit of the other party. We also see that this assumption puts a lower limit on the value of $p_{x y}$, while the original assumption of Brown $\left(p_{x y}<0\right)$ puts an upper limit. Therefore, the two assumptions combined determine a range of allowable values of $p_{x y}$ for the results of this paper to hold. 
The question arises whether these assumptions are realistic. It is indeed easy to see that Brown's assumption, $\left(p_{x y}<0\right)$, is not valid for a wide range of probability distributions where the injurer's and victim's precautions act independently of each other. Mathematically, if $p(x, y)$ can be written as a product of two independent probability functions $q^{1}(x)$ and $q^{2}(y)$ :

$$
p(x, y)=q^{1}(x) \cdot q^{2}(y)
$$

And if the first derivatives, $p_{x}$ and $p_{y}$ are always positive, then $p_{x y}$ will also be positive and so Brown's assumption does not hold. (It may, however, be noted that the assumptions in Eqs. (5) and (6) hold good even in this case)

As an example, in all the cases discussed in Guttel (2007), the marginal benefits of any of the precautions of one party do not depend on the precautions taken by the other party. Thus, $p_{x y}$ in these cases is equal to (or greater than) zero and therefore Brown's assumption would not hold.

However, it is to be understood that these examples have been designed to illustrate a concept and may not be representative of realistic situations. Brown's assumption of $p_{x y}<0$, on the other hand, is based on the sound principle of diminishing returns which, though not followed by many abstract mathematical functions, is inevitably seen to hold in all real life economic situations. Similarly, our assumptions in Eqs. (5) and (6) are based on the reasonable assumption that in ordinary situations an additional precaution by one party shall have a more direct impact on its own marginal benefits (as it has exhausted one possible precaution) than on the marginal benefits of the other party. We can therefore rest assured that the assumptions made in this paper would hold good in most, if not all, real life situations.

\section{Appendix II}

\section{A Comment on the Liability Rule of Negligence with Restitution of Cost Justified Precaution}

Guttel in his paper (Guttel 2007) makes a novel suggestion that an efficient outcome can be achieved if the determination of standard of care using the principle of cost justified untaken precaution is combined with restitution for cost justified precautions.

It must be mentioned here that the examples cited by Guttel in his paper are not directly amenable to an analysis using Brown's model. In those examples, abatement of harm requires acts by both parties, i.e. an act by one party alone would not lead to any reduction in probability of harm. On the contrary, under 
Brown's model, all acts of either party reduce the probability of harm. A proper analysis of the examples cited by Guttel requires an extended mathematical model. In this part, however, we limit ourselves to the question of whether his proposed liability rule of negligence with restitution for cost justified precautions can lead to an efficient outcome under Brown's model.

\section{Analysis of Strategy of Injured and Injurer}

Presuming that both the parties adopt socially optimal levels of precaution $\left(X^{\star}, Y^{\star}\right)$, the injurer has no cost justified untaken precaution and at the same time, the last precaution taken is cost justified. Hence, at this level, the injurer bears no cost (not even her own cost of precaution). Any increase in $X$ would make her last precaution cost unjustified (because $p_{x x}<0$ ) and hence, she would have to bear her precaution costs. A decrease in $X$ would allow her to claim restitution, but the cost of harm will stand shifted to him. Hence, injurer has no incentive to move away from the optimal level.

In the case of injured, a decrease in $Y$ from the optimal level of precaution makes the untaken precautions of injurer cost justified (because $p_{x y}<0$ ) and therefore the harm costs are shifted to the injurer. Thus, there is an incentive for the injured to move away from the optimal level of precaution

So, the liability rule of negligence with restitution of cost justified precaution does not have a Nash equilibrium at the optimum level of precaution, under Brown's model. In addition, as already stated in the main paper, this method requires courts to inquire into all precautions already taken by either party, which goes well beyond the 'limited information' regime described by Brown.

\section{References}

Assaf, G.B. (1984). The shape of reaction functions and efficiency of liability rules: a correction. J. Leg. Stud. 13: 101-111.

Brown, J.P. (1973). Towards an economic theory of liability. J. Leg. Stud. 2: 323-349.

Calabresi, G. (1970). The costs of accidents-a legal and economic analysis. Yale University Press, New Haven and London.

Cooter, R., Lewis, K., and Lane, D. (1979). Liability rules, limited information and the role of precedent. Bell J. Econ. 10: 366-373.

Diamond, P.A. (1974). Single activity accidents. J. Leg. Stud. 3: 107-164.

Grady, M.F. (1989). Untaken precautions. J. Leg. Stud. 18: 138-156.

Green, J. (1976). On the optimal structure of liability laws. Bell J. Econ. 7: 553-574.

Guttel, E. (2007). The (Hidden) risk of opportunistic precautions. UC Berkeley, Law and Economics Workshop, Available at: https://escholarship.org/content/qt4wd626rz/qt4wd626rz.pdf (Accessed 16 February 2020). 
Jain, S.K. (2006). Efficiency of liability rules: a reconsideration. J. Int. Trade Econ. Dev. 15: 359-373. Jain, S.K. and Singh, R. (2002). Efficient liability rules: complete characterisation. J. Econ. 75: $105-124$.

Jain, S.K. 2009. On the efficiency of negligence rule, Available at: https://doi.org/10.2139/ssrn. 1436588 (Accessed 16 February 2020).

Ott, C. and Schafer, H.-B. (1997). Negligence as untaken precaution, limited informationand efficient standard formation in the civil liability system. Int. Rev. Law Econ. 17: 15-29.

Rose-Ackerman, S. (1991). Regulation and the law of torts. Am. Econ. Rev. 81: 54-58, https://www. jstor.org/stable/2006825 (Accessed 7 November 2020).

Rubinfeld, D.L. (1987). The efficiency of comparative negligence. J. Leg. Stud. 16: 375-394.

Shavell, S. and Kaplow, L. (1996). Accuracy in the assessment of damages. J. Law Econ. 39: 191-210.

Shavell, S. and Kaplow, L. (1994). Accuracy in the determination of liability. J. Law Econ. 37: 1-15.

Shavell, S. (2013). A fundamental enforcement cost advantage of the negligence rule over regulation. J. Leg. Stud. 42: 275-302. 